\title{
Criterion validity of a 10-category scale for ranking physical activity in Norwegian women
}

Kristin B Borch ${ }^{1 *}$, Ulf Ekelund ${ }^{2}$, Søren Brage ${ }^{2}$ and Eiliv Lund ${ }^{1}$

\begin{abstract}
Background: Accurate measurement of physical activity (PA) is critical to establish dose-response relationships with various health outcomes. We compared the self-administered PA questionnaire from the Norwegian Women and Cancer Study (NOWAC) with a criterion method in middle-aged Norwegian women.

Methods: A sample of 177 randomly recruited healthy women attended two clinical visits approximately 4-6 months apart. At each visit, the women completed the NOWAC PA questionnaire (NOPAQ), rating their overall PA level on a 10-category scale (1 being a "very low" and 10 being a "very high" PA level) and performed an 8-minute step-test to estimate aerobic fitness $\left(\mathrm{VO}_{2} \mathrm{max}\right)$. After each visit, the women wore a combined heart rate and movement sensor for 4 consecutive days of free-living. Measures of PA obtained from the combined heart rate and movement sensor, which were used as criterion, included individually calibrated PA energy expenditure (PAEE), acceleration, and hours/day of moderate-to-vigorous intensity PA (MVPA). These were averaged between visits and compared to NOPAQ scores at visit 2.

Results: Intra-class correlation coefficients for objective measures from both free-living periods were in the range of $0.65-0.87(P<0.001)$, compared to $0.62(P<0.001)$ for NOPAQ. There was a moderate but significant $(P<0.001)$ Spearman's rank correlation coefficient in the range of 0.36-0.46 between NOPAQ and objective measures of PA. Linear trends for the association between the NOPAQ rating scale with PAEE, hours/day of MVPA and $\mathrm{VO}_{2}$ max $(P<$ 0.001) were also demonstrated.
\end{abstract}

Conclusions: Self-reported PA level measured on a 10-category scale appears valid to rank PA in a female Norwegian population.

Keywords: Accelerometry, combined sensor, heart rate, physical activity, self-report, validation

\section{Background}

In large-scale epidemiologic studies, physical activity (PA) is often assessed using questionnaires [1,2]. Selfreport methods as global questionnaires are commonly used to assess the relationship with health outcomes in order to rank or classify individuals as either physically active or inactive [3,4]. Indeed, a number of different PA questionnaires have been developed for various purposes such as surveillance, etiological investigation and risk stratification [5]. PA is a complex multidimensional behavior characterized in terms of volume, domain, type, duration, intensity and frequency [6], which makes PA inherently difficult to assess accurately in epidemiologic

\footnotetext{
* Correspondence: kristin.benjaminsen.borch@uit.no

'Department of Community Medicine, University of Tromsø, NORWAY

Full list of author information is available at the end of the article
}

studies. Particularly challenging is the estimation of PA energy expenditure (PAEE) [4,7]. Nonetheless, documentation of a questionnaire's precision is important to interpret the information it provides. Independent criterion methods that can accurately assess PAEE is key when examining the validity of PA questionnaires that aim to estimate PAEE as an integrated measure of self-reported duration, intensity and frequency of PA [3]. The gold standard for measuring PAEE during free-living conditions is the doubly-labeled water method, combined with an assessment of resting metabolic rate. However, this approach is expensive and does not provide any information on intensity and frequency patterns. Therefore, the most commonly used measures are derived from accelerometry, heart rate monitoring, or combined heart rate and movement sensing.
C Biomed Central

() 2012 Borch et al; licensee BioMed Central Ltd. This is an Open Access article distributed under the terms of the Creative Commons Attribution License (http://creativecommons.org/licenses/by/2.0), which permits unrestricted use, distribution, and reproduction in any medium, provided the original work is properly cited. 
The Norwegian Women and Cancer (NOWAC) Study is a population-based, nation-wide cohort study that was initiated in 1991 [8]. The study was originally set up to estimate the risk of breast cancer and its association with oral contraceptive use, and has since been expanded to examine the possible association between several exposures and different types of cancer and other chronic diseases [8]. The NOWAC PA questionnaire (NOPAQ) is a unique and simple self-report instrument for ranking of PA level using a 10-category scale. It was mainly developed to enable adjustment for PA as a confounding variable when examining associations between other healthrelated exposures and disease. PA exposure as assessed by the NOPAQ has not been previously validated. We therefore aimed to evaluate the criterion-related validity of the NOPAQ against objective estimates of PA, as assessed by individually-calibrated combined heart rate $(\mathrm{HR})$ and movement sensoring. In addition, we examined the influence of aerobic fitness $\left(\mathrm{VO}_{2} \mathrm{max}\right)$. The results from this study will provide useful knowledge regarding PA assessment methodology within a female population.

\section{Methods}

\section{Study Design}

The study was carried out from March 2007 to March 2008 , covering all four seasons. It consisted of two clinical visits, approximately 4 to 6 months apart (mean time between visits $=4.99$ (0.92 months). Each clinical visit included completion of the NOPAQ and an 8-minute step-test. Each clinical visit was followed by combined heart rate (HR) and movement monitoring for at least 4 consecutive days and nights of free-living. This study followed the protocol of The InterAct validation study [9], which aimed to validate different PA questionnaires within the European Prospective Investigation into Nutrition and Cancer, of which the NOWAC study is part.

\section{Study population}

Troms $\varnothing$ is a city located at latitude $69^{\circ} \mathrm{N}$ in the county of Troms, an area characterized by large seasonal variations. A random sample of 600 women between 40-55 years of age and living in Tromsø was drawn in 2007 from the National Population Registry, Statistics Norway. Due to emigration and unknown addresses, 589 women were invited to participate in this study. The participants had to live in the same municipality as the investigation premises at the University of Tromsø to match the original sample in the NOWAC study [8]. Exclusion criteria were conditions that had led to mobility limitations, which made walking unaided impossible. A total of 221 women agreed to participate; however 23 women did not come to the first clinical visit, resulting in an initial study sample of 198 women (overall response rate 33.6\%). Following the two clinical visits complete data was available for 177 women: 4 did not provide sufficient free-living data at visit 1 and a further 17 had missing data at visit 2 (Figure 1). Participants taking medications that affected heart rate (use of beta blockers, $50 \%$ or more of maximum dose, $n=$ 1) were excluded from the step-test, but were included in all other parts of the study. Written informed consent was obtained from each participant and ethical approval for the study was obtained from the Regional Committee for Medical Research Ethics, North of Norway, and the Norwegian Data Inspectorate.

\section{Anthropometrics}

Height (to the nearest $\mathrm{mm}$ ) and weight (to the nearest $0.1 \mathrm{~kg}$ ) were measured by a Seca 764 electronic body height and weight measuring instrument (Seca gmbh \& co.kg, Hamburg, Germany). Both were measured with the participant in light clothing and without shoes. Body mass index was calculated as weight in kilograms divided by height in meters squared $\left(\mathrm{kg} / \mathrm{m}^{2}\right)$, and categorized into three levels; normal $\left(<25 \mathrm{~kg} / \mathrm{m}^{2}\right)$, overweight $\left(25-30 \mathrm{~kg} / \mathrm{m}^{2}\right)$ and obese $\left(>30 \mathrm{~kg} / \mathrm{m}^{2}\right)$.

\section{Measures of PA \\ Objective measures of PA and fitness}

Eligible participants were fitted with a combined heart rate and movement sensor (Actiheart, CamNtech, Cambridge, UK), which was attached to the chest via two standard electrocardiogram electrodes [10].

Study participants performed an 8-minute ramped steptest using a 200-mm step (Reebok, Lancaster, UK) to determine the individual relationship between heart rate and workload [11]. Specifically, participants were asked to step up and down following a timed voice prompt at a step frequency that began at 15 body lifts per minute (60 steps/min) and increased linearly to a maximum of 33 lifts per minute, immediately followed by a 2-minute seated recovery phase. In addition to their utility for individual calibration of free-living heart rate data, the parameters of the step test were used to estimate $\mathrm{VO}_{2} \max (\mathrm{mL} / \mathrm{kg} / \mathrm{min})$ by extrapolating each individual's sub-maximal HR-PAEE relationship to age-predicted maximum HR [12] and adding an estimate of resting metabolic rate [13].

Following each step-test, the combined heart rate and movement sensor was downloaded and re-initialized for long-term recording on a 1-minute epoch and participants were instructed to wear the monitor continuously for a minimum of 4 consecutive days and nights. Data collected during these free-living periods was downloaded to a computer database and the heart rate trace was processed using a robust Gaussian Process regression method to handle potential measurement noise [14]. PA intensity $(\mathrm{J} / \mathrm{min} / \mathrm{kg})$ for each time point was estimated from the combination of individually calibrated heart rate and movement data [11] using a branched equation 


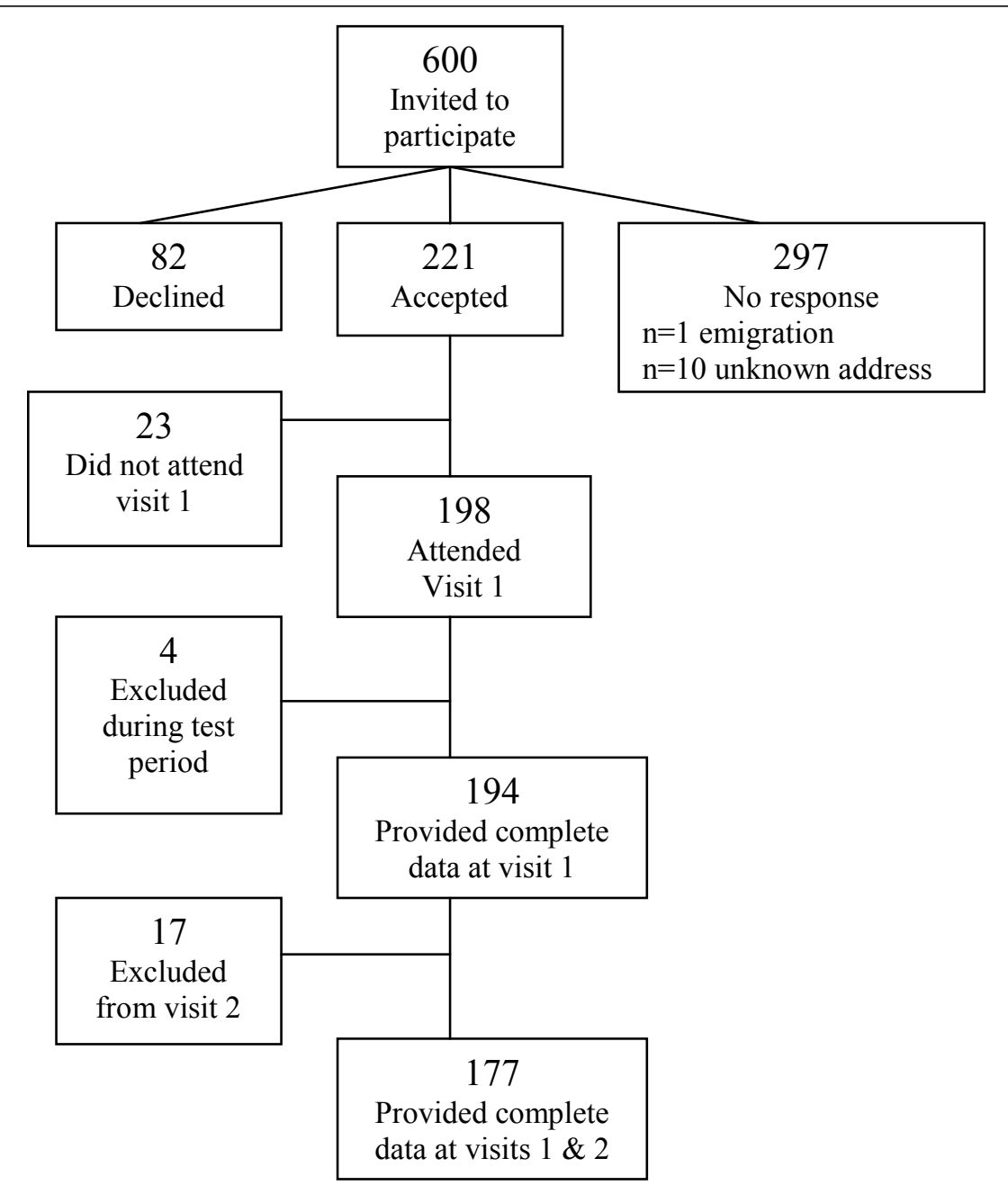

Figure 1 Flow chart - inclusion of the study participants.

framework [15]. Periods of non-wear were inferred from the combination of non-physiological heart rate and prolonged periods of inactivity, which were taken into account to minimize diurnal information bias when summarizing the intensity time-series into PAEE $(\mathrm{kJ} / \mathrm{kg} / \mathrm{day})$, hours per day of light intensity PA $(<3$ metabolic equivalent of task units, MET), moderate-to-vigorous PA (MVPA, > $3 \mathrm{MET}$ ), and average acceleration $\left(\mathrm{m} / \mathrm{s}^{2}\right)$.

\section{$P A$ questionnaire (NOPAQ)}

Study participants completed the NOPAQ at both clinical visits. The questionnaire asks the participant to rate their PA level at age 14 and 30 years, as well as "today" (the time of completion) on a scale of 1 to 10 , where 1 corresponds to a "very low" PA level and 10 corresponds to a "very high" PA level. PA was described in the questionnaire as "By physical activity we mean both work in and outside the home, as well as training/exercise and other physical activity, such as walking, etc. Mark the number that best describes your level of physical activity." (See additional file 1 showing questionnaire excerpt). The response given for the PA level at the time of questionnaire completion ("today") was evaluated in this validation study.

\section{Statistical analysis}

Descriptive data (age, height, weight, body mass index, fitness, and objective physical activity measures) were first summarized on an individual level based on measures from both clinical visits and the sample mean \pm standard deviation (SD) was then reported. Outcomes from the NOPAQ were described with frequency distribution. Responses from the NOPAQ completed at visit 2 were used in the comparison with objective measures. Relative agreement between visit 1 and visit 2 measures were examined by Intra-class correlation coefficients (ICC). Time between assessments did not influence these results, so they were left unadjusted. Criterion validity of the NOPAQ against objective measures (PAEE, accelerometry, 
MVPA and $\mathrm{VO}_{2} \max$ ) was examined by Spearman's rank correlation coefficient $(\sigma)$. The NOPAQ was analyzed using each level of the 10-category scale as a categorical variable in correlation analyses. Since participants may also take their perceived fitness level into account when self-reporting their PA level, we also combined the $\mathrm{VO}_{2}$ max estimate and the PAEE estimate into a new variable by averaging their z-scores (zPAFIT), and compared with the NOPAQ. Linear regression analysis was used to examine relationships between the PAEE and zPAFIT measures and the NOPAQ (self-reported PA level treated as a continuous variable) at three levels of adjustment; unadjusted, age and BMI adjusted, and with additional adjustment for VO2max (PAEE model only). Assumptions of linear regression analysis (i.e., normally distributed residuals) was examined by the Shapiro-Wilk W test. For all analyses, the level of significance was set at $P<0.05$. STATA version 11.0, special edition (StataCorp, College Station, TX, USA) was used for the statistical analyses.

\section{Results}

\section{Characteristics of the study participants and PA level}

Table 1 presents descriptive characteristics of study participants. The median self-reported PA level was 5 on the 10 -category scale, with $41.2 \%$ of participants ranking their PA level as 5 or $6,28.3 \%$ as 1 to 4 and $30.5 \%$ ranking their PA level as 7 or higher. The participants spent on average $41 \%$ of their time at sedentary $(\leq 1 \mathrm{MET})$ and $53 \%$ at low (1-3 METs) PA intensity according to combined sensor measures of PA. The monitor was worn by participants for an average of 4.7 (0.22) days and nights during each of the two measurement periods.

Table 2 displays the ICC for PAEE, acceleration, MVPA, $\mathrm{VO}_{2}$ max, zPAFIT and the NOPAQ (all $\mathrm{P}<$ 0.001). It indicates a moderate agreement for all activity measures, and similar between objective and self-report

Table 1 Descriptive characteristics of study participants, $\mathrm{n}=177$ women

\begin{tabular}{|c|c|c|c|c|}
\hline Characteristics & Mean & SD & Min & Max \\
\hline Age (years) & 47.5 & 4.4 & 40 & 55 \\
\hline Height (m) & 1.7 & 0.06 & 1.51 & 1.8 \\
\hline Weight (kg) & 71.1 & 10.9 & 47 & 115.3 \\
\hline BMI $\left(\mathrm{kg} / \mathrm{m}^{2}\right)$ & 26.1 & 3.5 & 18.8 & 37.5 \\
\hline PAEE (kJ/kg/day) & 44.9 & 14.4 & 17.5 & 96.6 \\
\hline Acceleration $\left(\mathrm{m} / \mathrm{s}^{2}\right)$ & 0.1 & 0.04 & 0.02 & 0.3 \\
\hline Sedentary time (hours/day) & 9.9 & 1.4 & 6.6 & 15.4 \\
\hline Low intensity PA (hours/day) & 12.6 & 1.4 & 7.5 & 16.0 \\
\hline MVPA (hours/day) & 1.5 & 0.9 & 0.2 & 6.5 \\
\hline $\mathrm{VO}_{2} \max \left(\mathrm{mlO}_{2} / \mathrm{min} / \mathrm{kg}\right)$ & 31.9 & 4.8 & 22.8 & 53.9 \\
\hline NOPAQ* & 5 & 3 & 1 & 10 \\
\hline
\end{tabular}

*Self-reported PA level median (interquartile range)
Table 2 Relative agreement between measures obtained from the two visits

\begin{tabular}{lll}
\hline Outcome & $\mathrm{ICC}^{*}$ & $\mathbf{9 5 \% \mathrm { Cl } ^ { \dagger }}$ \\
\hline PAEE & 0.65 & {$[0.56,0.74]$} \\
MVPA & 0.66 & {$[0.56,0.74]$} \\
Acceleration & 0.65 & {$[0.56,0.73]$} \\
VO $_{2}$ max & 0.87 & {$[0.81,0.89]$} \\
zPAFIT & 0.84 & {$[0.79,0.88]$} \\
NOPAQ & 0.62 & {$[0.52,0.71]$} \\
\hline
\end{tabular}

*Intraclass correlation coefficient

${ }^{\dagger}$ Confidence interval

measures. $\mathrm{VO}_{2} \max$ measures at first and second clinical visit were more strongly correlated, with an ICC of 0.87 .

Comparing self-reported PA level and objective measures Sensor-based measures of PA and fitness were stratified by self-reported PA levels (Table 3). Overall, significant linear trends of PAEE, acceleration, MVPA, $\mathrm{VO}_{2} \max$ and zPAFIT across self-reported PA levels were observed. Self-reported PA level was positively associated with level of PAEE, and a trend of increasing acceleration from the lowest to the highest self-reported PA level was also observed. Higher self-reported PA level was associated with higher durations of MVPA (> 3 METs). Similarly, those who characterized themselves as less active accumulated less MVPA. Furthermore, higher self-reported PA levels were associated with higher $\mathrm{VO}_{2}$ max estimates and lower body mass index.

The associations between the NOPAQ and criterion measures were significantly correlated, with Spearman's rank correlation coefficients of 0.36-0.46, depending on the criterion measure: PAEE $(\sigma=0.39)$, MVPA $(\sigma=0.42)$, $\mathrm{VO}_{2} \max (\sigma=0.36)$ and zPAFIT $(\sigma=0.41)$. The strongest correlation was observed for accelerometry $(\sigma=0.46)$.

Relationships between the NOPAQ and the criterion measures PAEE and zPAFIT were examined by linear regression analysis (Table 4). Self-reported PA level contributed significantly to explain variance in both PAEE and zPAFIT at all levels of adjustment.

\section{Discussion}

The present study examined the criterion validity of a simple self-reported ranking instrument, the NOPAQ, which was aimed at ranking overall PA level in Norwegian women aged 40-55 years. In general, the results showed significant but moderate Spearman's rank correlation coefficients in the range of 0.36-0.46, and linear trends in the relationship between self-reported PA level and objective measures of PA derived from registration of heart rate and movement during free-living, including PAEE, acceleration, and MVPA. In addition, NOPAQ 
Table 3 Objective measures of physical activity and fitness by self-reported PA level among 177 women

\begin{tabular}{llllllll}
\hline PA level & \% PA (N) & PAEE & MVPA & Acceleration & VO $_{\mathbf{2}}$ max & zPAFIT & BMI \\
\hline $\mathbf{1}$ & $1.7(3)$ & $31.8(4.50)$ & $.80(.10)$ & $.07(.003)$ & $29.1(1.10)$ & $-.70(.33)$ & $29.1(2.68)$ \\
$\mathbf{2}$ & $5.1(9)$ & $35.7(3.17)$ & $.87(.19)$ & $.08(.006)$ & $29.9(.95)$ & $-.42(.19)$ & $25.0(1.16)$ \\
$\mathbf{3}$ & $7.4(13)$ & $37.7(5.01)$ & $1.16(.31)$ & $.08(.006)$ & $22.4(.89)$ & $-.59(.27)$ & $27.5(.96)$ \\
$\mathbf{4}$ & $14.1(25)$ & $39.3(2.07)$ & $1.31(.15)$ & $.09(.007)$ & $29.9(.67)$ & $-.43(.11)$ & $27.4(.72)$ \\
$\mathbf{5}$ & $23.2(41)$ & $42.4(1.97)$ & $1.38(.10)$ & $.11(.006)$ & $31.6(.91)$ & $-.13(.15)$ & $26.7(.60)$ \\
$\mathbf{6}$ & $18.1(32)$ & $47.7(2.62)$ & $1.49(.13)$ & $.13(.009)$ & $33.4(.65)$ & $.35(.13)$ & $24.1(.53)$ \\
$\mathbf{7}$ & $17.5(31)$ & $52.1(2.43)$ & $1.98(.15)$ & $.12(.006)$ & $33.8(.84)$ & $.32(.14)$ & $26.4(.60)$ \\
$\mathbf{8}$ & $10.2(18)$ & $47.7(2.39)$ & $1.67(.14)$ & $.14(.008)$ & $32.3(.70)$ & $.10(.12)$ & $25.7(.63)$ \\
$\mathbf{9}$ & $1.1(2)$ & $57.1(17.91)$ & $2.44(.1 .30)$ & $.13(.01)$ & $29.7(3.72)$ & $.12(.81)$ & $25.0(.81)$ \\
$\mathbf{1 0}$ & $1.7(3)$ & $70.4(14.40)$ & $3.42(1.58)$ & $.16(.04)$ & $41.1(7.15)$ & $1.84(1.14)$ & $25.1(1.86)$ \\
\hline Test for trend & & $P<0.001$ & $P<0.001$ & $P<0.001$ & $P<0.001$ & $P<0.001$ & $P=0.047$ \\
\hline
\end{tabular}

correlates positively with $\mathrm{VO}_{2} \max$. The results also showed that stability statistics were comparable for both objective and subjective activity measures over a period of 4-6 months, as assessed using ICCs (0.62-0.65). Taking into account that PA is a modifiable behavior, this indicates that physical activity level of middle-aged women may be relatively stable over a time period of 4 to 6 months, and across large seasonal variations in Norway, although not as stable as fitness.

Our results are in agreement with previous studies examining the validity of other self-reported ranking instruments to measure global PA. In a review [16] on self-reported PA questionnaires comparing different objective measures of PA (e.g., doubly-labeled water method, accelerometry, and HR monitoring) with self-reported PA, the correlation coefficients ranged between 0.14-0.36. More complex questionnaires have been compared with relatively simple measures of global PA and correlation coefficients were reported in the range of 0.14-0.41 [17]. Long and complex PA questionnaires may be demanding for the respondents and may therefore explain why some of the highest coefficients for reliability and validity are seen for global PA questionnaires [17]. A validation study of the International Physical Activity Questionnaire against accelerometry across 12 countries reported a

Table 4 Relationship between self-report and objective measures of physical activity

\begin{tabular}{lcccccc}
\hline & \multicolumn{3}{c}{ PAEE } & \multicolumn{3}{c}{ zPAFIT } \\
\cline { 2 - 7 } Model & $\boldsymbol{\beta}$ & $\mathbf{9 5 \%} \mathbf{C l}$ & $\mathbf{R 2}$ & $\boldsymbol{\beta}$ & $\mathbf{9 5 \%} \mathbf{C l}$ & $\mathbf{R 2}$ \\
\hline NOPAQ & 0.049 & {$[0.029$,} & 0.12 & 0.18 & {$[0.12$,} & 0.15 \\
& & $0.069]$ & & & $0.26]$ & \\
NOPAQ & 0.039 & {$[0.021$,} & 0.34 & 0.15 & {$[0.09$,} & 0.34 \\
(W/BMl and age) & & $0.056]$ & & & $0.21]$ & \\
NOPAQ & 0.021 & {$[0.005$,} & 0.48 & & & \\
(W/BMl, age, and & & $0.038]$ & & & & \\
VO ${ }_{2}$ max) & & & & & & \\
\hline
\end{tabular}

The $\beta$-coefficient denotes the difference in the outcome variable by a 1-unit difference in the NOPAQ scale. $\mathrm{Cl}=$ Confidence Interval. $\mathrm{R} 2=$ model explained variance criterion validity by Spearman correlation with a median of 0.30 [18]. It is interesting that the simple NOPAQ ranking instrument correlates quite strongly with the criterion measures, even higher than some PA questionnaires that aim to measure different dimensions of PA. However, an alternative explanation is that the lower correlations observed in other studies are due to limitations of the criterion method used for comparison; even though the correlation with accelerometry in our study was also high, this criterion measure may have been stronger in our study since the participants wore the monitor 24 hours a day during two 4-day observation periods. We cannot however compare correlations directly to ascertain which self-reported instrument may be superior. One exception is the recent report by the InterAct Consortium on validity of the short EPIC questionnaire, carried out in 10 European countries and including the present sample of Norwegian women [9]. This study, using the same criterion measures and sampling protocols, reported Spearman correlations of 0.32 and 0.35 in the Norwegian sample for the best indices derived from that questionnaire, which is almost identical to the NOPAQ result. The acceptable measurement properties of the NOPAQ compared to other more established self-reported instruments could be due to the simplicity of its design. It may be easier for the participant to answer questions on PA when only one general dimension of PA is sought [19]. The NOPAQ includes several dimensions collapsed into one global PA measure, and could be considered beneficial. To this end, face validity of the NOPAQ was recently demonstrated in a followup within the original NOWAC study, in that PA level was predictive of mortality and also inversely associated with BMI [20].

Strengths of the present study include the criterion method of individually calibrated HR and movement sensing in a repeated measurement study design. Using multiple measures will most likely provide a more robust estimate of "true" PA, as compared to using a single measure. Apart from the doubly-labeled water 
method, combined HR and movement sensoring is probably the most accurate criterion method available from which to derive PAEE measures [5]. Indeed, a combined sensor that provides estimates of PAEE using accelerometry and heart rate monitoring data may overcome some of the limitations associated using these methods separately. Accelerometry, for example, is limited for assessing PAEE of certain types of activities such as cycling and carrying heavy loads. Heart rate monitoring is limited when measuring low-intensity activity due to stronger relative influence of stress and relies more heavily on individual calibration [15]. A combined monitor with individual calibration of the heart rate-PAEE relationship and branched modeling for combining the physiological and biomechanical information recorded has been shown to reduce the error of predicted PAEE [11]. The criterion measure of PA intensity and its time-integral, PAEE, has been successfully validated against indirect calorimetry during simulated daily living activities [21-23] and during free-living against the doubly-labeled water method [24].

Between 3 and 5 days is usually considered appropriate for providing reliable measures of free-living PA by accelerometry [25]. In this study, the participants wore the monitors for $>4$ days including nights after each clinical visit, which is also considered sufficient for obtaining reliable PAEE estimates. This protocol also provided data on sleeping heart rate which, in addition to aiding individual calibration, improved the precision of PAEE estimates [15]. Another important strength of this study includes a 24-hour protocol which makes non-wear time less of an issue. The monitors were given to participants during a face-to-face clinical visit and this approach made certain that care and use of the monitor was explained adequately.

Our results also indicated that higher $\mathrm{VO}_{2}$ max was associated with higher self-reported PA level. It is a general understanding among many people that fitness $\left(\mathrm{VO}_{2} \mathrm{max}\right)$ is the same as PA [26], despite the fact that the constructs are different, one being a capacity ("can do") quantity and the other being a behavioral ("will do") quantity. It is also argued that most people have a fairly clear perception of their fitness level according to their leisure time PA levels [17] but to generalize such predictions is perhaps questionable. To correct for the possibility that participants may take into account their perceived fitness level when reporting their PA level and the resulting bias, the association with PAEE was adjusted for $\mathrm{VO}_{2}$ max in the linear regression analysis, in addition to examining a composite outcome of PAEE and $\mathrm{VO}_{2} \max$ in the zPAFIT variable. The magnitude of the association between NOPAQ and PAEE was attenuated but still significant after adjusting for fitness. In addition, the zPAFIT variable showed only minor improvement in the correlation with self-reported
PA level, which suggests that the NOPAQ does not suffer severely from any bias by $\mathrm{VO}_{2}$ max level.

The participants in the present study were representative of the population of the NOWAC Study in terms of age and sex. The NOWAC study population is further considered representative of Norwegian middle-aged women [8]. Nonetheless, it is important to highlight that examining agreement between instruments done in one group may not generalize to others. The NOPAQ was completed twice, at two separate clinical visits by participants in this study, and an ICC of 0.62 indicated a moderate agreement; the responses did not differ substantially over a 4-6 month time period. The participants live in an area with considerable seasonal variations, which could explain some of the differences in self-reported PA level between visits. This is supported by the fact that ICCs were similar for sensor-based measures of PA.

The main advantages of a PA questionnaire such as the NOPAQ that uses an ordinal scale are the ease of design, administration, and data handling. Nevertheless, there are other challenges associated with the use of a simple rating scale. Studies have demonstrated that self-reported PA is usually overestimated when compared with criterion measures of PA $[27,28]$. Calibrating highly subjective experiences such as perceived total amount of PA along a continuum of equal intervals is difficult. Assessment of a subjective phenomenon will always be a challenge considering the fact that categorizing and quantification never will totally embrace a phenomenon [29]. Another criticism of an ordinal scale is the reluctance of subjects to make full use of its range, preferring to avoid extreme responses [30]. A numeric scale will obviously raise difficulties for some individuals in rating a self-perceived behavior like PA, and reference frames will probably differ widely. In order to use a scale well, respondents need a reference frame when choosing PA levels [31]. The challenge with the NOPAQ is that it uses a numeric scale to rank PA levels in individuals. It could be difficult for the respondent to differentiate between and interpret the 10 levels without a reference frame to associate with the various levels of the scale. Both vigorous PA and sedentary behavior are two important sub-dimensions of PA related to health [32]. Our results suggest that the NOPAQ is sufficient to rank individuals into categories of global PA level which is of relevant usability. However, the scale does not give insight into the type, frequency, intensity, or duration of PA, nor the domains in which PA takes place; these characteristics limits the utility of the NOPAQ. A limitation of the present study was that there were fewer individuals in the lower and higher selfreported PA categories. However, we would not expect many women between 40 and 55 years to be categorized into either of these groups, and we found the study 
population normally distributed according to selfreported PA level.

The fact that correlation coefficients are significant does not automatically imply that the two measures are identical and the results should be interpreted accordingly. Both questionnaire- and sensor-based measures of activity clearly assess PA differently, each with individual strengths and limitations.

It is important to maintain consistency in surveillance of PA in a prospective cohort study like the NOWAC Study, and proceed with the same PA questionnaire for future follow-up. However, one should consider an alternative PA questionnaire when it comes to quantifying PA level in different domains and types, as well as the duration, intensity and frequency that make up the total volume of PA and help to understand the mechanisms of PA and its relation to different diseases. As for the continued use of the NOPAQ in the NOWAC Study, results from our present report are encouraging and demonstrate the criterion-related validity of the measure used to define PA level in this population.

\section{Conclusions}

In summary, the NOPAQ was found to be valid, with modest correlations compared with criterion measures, making this self-report instrument suitable to differentiate general PA levels in an adult female population in Norway.

\section{Additional material}

Additional file 1: The Norwegian Women and Cancer Study questionnaire on physical activity (NOPAQ).

\section{Acknowledgements}

This study was supported by funding from the Medical Research Council Epidemiology Unit, Institute of Metabolic Science, Cambridge, UK.

\section{Author details}

${ }^{1}$ Department of Community Medicine, University of Tromsø, NORWAY. ${ }^{2}$ Medical Research Council Epidemiology Unit, Institute of Metabolic Science, Cambridge, England, UK.

\section{Authors' contributions}

KBB collected data, carried out the statistical analysis and drafted the manuscript. UE has made substantial contributions to conception and design and has contributed with critical revision of the manuscript. SB designed and derived all objective measures of activity and fitness, contributed to the statistical analysis and interpretation of the data and critical revision of the manuscript. EL is the principal investigator and designed the NOWAC Study, and contributed with critical revision of the manuscript. All authors read and approved the final manuscript.

\section{Competing interests}

The authors declare that they have no competing interests.

Received: 27 June 2011 Accepted: 19 January 2012 Published: 19 January 2012
References

1. Corder K, Ekelund U, Steele RM, Wareham NJ, Brage S: Assessment of physical activity in youth. J Appl Physiol 2008, 105:977-987.

2. Warren JM, Ekelund U, Besson H, Mezzani A, Geladas N, Vanhees L: Assessment of physical activity - a review of methodologies with reference to epidemiological research: a report of the exercise physiology section of the European Association of Cardiovascular Prevention and Rehabilitation. European Journal of Cardiovascular Prevention \& Rehabilitation 2010, 17:127-139.

3. Epidemiologic Methods in Physical Activity Studies. Edited by: Lee I-M, Blair SN, Manson JAE, Paffenbarger RS Jr. New York: Oxford University Press; 2009:

4. Lamonte MJ, Ainsworth BE: Quantifying energy expenditure and physical activity in the context of dose response. Med Sci Sports Exerc 2001, 33: \$370-\$378.

5. Besson H, Brage S, Jakes RW, Ekelund U, Wareham NJ: Estimating physical activity energy expenditure, sedentary time, and physical activity intensity by self-report in adults. Am J Clin Nutr 2010, 91:106-114.

6. Shephard RJ, Stephens T, Bouchard C: Physical activity, fitness, and health: international proceedings and consensus statement. c1994; Champaign, III Human Kinetics; 1994, XXIV, 1055 s.

7. Neilson HK, Robson PJ, Friedenreich CM, Csizmadi I: Estimating activity energy expenditure: how valid are physical activity questionnaires? Am J Clin Nutr 2008, 87:279-291.

8. Lund E, Dumeaux V, Braaten T, Hjartaker A, Engeset D, Skeie G, Kumle M: Cohort Profile: The Norwegian Women and Cancer Study-NOWACKvinner og kreft. Int J Epidemiol 2008, 37:36-41.

9. Peters T, Brage S, Westgate K, Franks PW, Diaz MJT, Bendinelli B, Huerta JM, Vigl M, Wendel-Vos W, Gradmark A, Borch KB, Guillain BL, Sharp S, Kerrison N, Langenberg C, Arriola L, Clavel-Chapelon F, Barricart A, Boeing H, Gonzales C, Kaaks R, Key T, Khaw KT, May A, Nilsson P, Norat T, Overvad K, Palli D, Panico S, Quiros R, et al: Validity of a short questionnaire to assess physical activity in 10 European countries. Eur $J$ Epidemiol

10. Brage S, Brage N, Franks PW, Ekelund U, Wareham NJ: Reliability and validity of the combined heart rate and movement sensor Actiheart. Eur J Clin Nutr 2005, 59:561-570.

11. Brage S, Ekelund U, Brage N, Hennings MA, Froberg K, Franks PW, Wareham NJ: Hierarchy of individual calibration levels for heart rate and accelerometry to measure physical activity. J Appl Physiol 2007, 103:682-692.

12. Tanaka H, Monahan KD, Seals DR: Age-predicted maximal heart rate revisited. Journal of the American College of Cardiology 2001, 37:153-156.

13. Henry C: Basal metabolic rate studies in humans: measurement and development of new equations. Public Health Nutrition 2005, 8:133-152

14. Stegle O, Fallert SV, MacKay DJ, Brage S: Gaussian Process Robust Regression for Noisy Heart Rate Data. Biomedical Engineering, IEEE Transactions on 2008, 55:2143-2151.

15. Brage $S$, Brage N, Franks PW, Ekelund U, Wong M-Y, Andersen LB, Froberg K, Wareham NJ: Branched equation modeling of simultaneous accelerometry and heart rate monitoring improves estimate of directly measured physical activity energy expenditure. J Appl Physiol 2004, 96:343-351.

16. Sallis JF, Saelens BE: Assessment of physical activity by self-report: Status, limitations, and future directions. Research Quarterly for Exercise and Sport 2000, 71:1.

17. Shephard R, Vuillemin A: Limits to the measurement of habitual physical activity by questionnaires. Br J Sports Med 2003, 37:197-206.

18. Craig CL, Marshall AL, Sjostrom M, Bauman AE, Booth ML, Ainsworth BE, Pratt M, Ekelund U, Yngve A, Sallis JF, Oja P: International Physical Activity Questionnaire: 12-Country Reliability and Validity. Med Sci Sports Exerc 2003, 35:1381-1395.

19. Kurtze N, Gundersen KT, Holmen J: Selvrapportert fysisk aktivitet i 1980og 1990 årene - Helseundersøkelsene i Nord-Trøndelag (HUNT). Norwegian Journal of Epidemiology 2003, 13:171-176.

20. Borch KB, Braaten T, Lund E, Weiderpass E: Physical activity and mortality among Norwegian women - the Norwegian Womwn and Cancer Study. Clinical Epidemiology 2011, 3:229-235.

21. Strath SJ, Brage S, Ekelund U: Integration of Physiological and Accelerometer Data to Improve Physical Activity Assessment. Medicine \& Science in Sports \& Exercise 2005, 37:S563-S571. 
22. Crouter S, Churilla J, Bassett D: Accuracy of the Actiheart for the assessment of energy expenditure in adults. European Journal of Clinical Nutrition 2008, 62:704.

23. Thompson D, Batterham AM, Bock S, Robson C, Stokes K: Assessment of Low-to-Moderate Intensity Physical Activity Thermogenesis in Young Adults Using Synchronized Heart Rate and Accelerometry with Branched-Equation Modeling. The Journal of Nutrition 2006, 136:1037-1043.

24. Assah FK, Ekelund U, Brage S, Wright A, Mbanya JC, Wareham NJ: Accuracy and validity of a combined heart rate and motion sensor for the measurement of free-living physical activity energy expenditure in adults in Cameroon. International Journal of Epidemiology 2011, 40:112-120.

25. Trost SG, LMK, RPR : Conducting Accelerometer-Based Activity Assessments in Field-Based Research. Medicine \& Science in Sports \& Exercise 2005, 37:S531-S543.

26. Aadahl $M$, Kjær $M$, Jørgensen T: Perceived exertion of physical activity: Negative association with self-rated fitness. Scandinavian Journal of Public Health 2007, 35:403-409.

27. Hagstromer M, Oja P, Sjostrom M: Physical Activity and Inactivity in an Adult Population Assessed by Accelerometry. Medicine \& Science in Sports \& Exercise 2007, 39:1502-1508

28. Troiano RP: A Timely Meeting: Objective Measurement of Physical Activity. Medicine \& Science in Sports \& Exercise 2005, 37:5487-S489.

29. Janz KF: Physical activity in epidemiology: moving from questionnaire to objective measurement. British Journal of Sports Medicine 2006, 40:191.

30. Livingstone MBE, Robson PJ, Welch RW, Burns AA, Burrows MS, McCormack C: Methodological issues in the assessment of satiety. Scandinavian Journal of Nutrition/Naringsforskning 2000, 44:98-103.

31. Sternfeld B, Cauley J, Harlow S, Liu G, Lee M: Assessment of Physical Activity with a Single Global Question in a Large, Multiethnic Sample of Midlife Women. Am J Epidemiol 2000, 152:678-687.

32. Physical Activity Guidelines Advisory Committee USDoH, Services H. 2008.

doi:10.1186/1479-5868-9-2

Cite this article as: Borch et al:: Criterion validity of a 10-category scale for ranking physical activity in Norwegian women. International Journal of Behavioral Nutrition and Physical Activity 2012 9:2.

\section{Submit your next manuscript to BioMed Central and take full advantage of:}

- Convenient online submission

- Thorough peer review

- No space constraints or color figure charges

- Immediate publication on acceptance

- Inclusion in PubMed, CAS, Scopus and Google Scholar

- Research which is freely available for redistribution

Submit your manuscript at www.biomedcentral.com/submit
Biomed Central 